\title{
Assessment of endogenous reference gene suitability for serum exosomal microRNA expression analysis in liver carcinoma resection studies
}

\author{
YI LI, GUI-MING XIANG, LIN-LIN LIU, CHANG LIU, FEI LIU, DONG-NENG JIANG and XIAO-YUN PU
}

Department of Clinical Laboratory, Xin Qiao Hospital, Third Military Medical University, Chongqing 400037, P.R. China

Received February 18, 2014; Accepted April 30, 2015

DOI: $10.3892 / \mathrm{mmr} .2015 .3919$

\begin{abstract}
Serum exosomal microRNAs (miRNAs) have received considerable attention as potential biomarkers for tumor diagnosis. Reverse transcription-quantitative polymerase chain reaction (RT-qPCR) is commonly used to detect miRNA expression levels in various types of cancer. One prerequisite for valid RT-qPCR data is the correct normalization of miRNAs to stably expressed endogenous reference genes (RGs). The study of liver carcinoma resection requires the use of reliable RGs in order to assess the expression levels of serum exosomal target miRNAs. However, the assessment of RG suitability for optimum serum exosomal miRNA expression analysis has yet to be investigated. The present study investigated the expression stability of 10 candidate RGs. The candidate genes included eight miRNAs (miR-16, miR-103, miR-191, let-7a, miR-26a, miR-221, miR-181a, and miR-451) and two small RNAs (5S and U6). The stability values of the candidate genes were calculated using the following algorithms: geNorm, NormFinder, BestKeeper, and the comparative $\Delta \mathrm{Ct}$ method. The overall ranking obtained from these analyses revealed that miR-221, let-7a, and miR-26a were appropriate internal RGs for analysis of serum miRNAs in patients with hepatocellular carcinoma. In addition, normalization with miR-221 and let-7a combined, as recommended by geNorm, or with miR-26a, as recommended by NormFinder, increased the accuracy of interpretation of the target miRNA expression levels in hepatopathy studies.
\end{abstract}

\section{Introduction}

MicroRNAs (miRNAs) are a small (18-21 bp) evolutionarily conserved subclass of RNA molecules that have important

Correspondence to: Professor Xiao-Yun Pu, Department of Clinical Laboratory, Xin Qiao Hospital, Third Military Medical University, 183 Xinqiao Street, Chongqing 400037, P.R. China E-mail: jyksci@163.com

Key words: reverse transcription quantitative polymerase chain reaction, serum exosomes, reference gene, microRNA, hepatocellular carcinoma roles in development, immunity, stem cell differentiation, and cancer (1,2). Exosomes are 40-100 nm membrane microvesicles of endocytic origin, which are released from various cell types under both normal and pathological conditions $(3,4)$. To date, exosomes have been identified in body fluids, including urine, amniotic fluid, malignant ascites, saliva, and blood (5-7).

Valadi et al (8) demonstrated that exosomes contain both mRNA and miRNA. A previous study also demonstrated that extracellular miRNA from exosomes has a role in cell-to-cell communication in hepatocellular carcinoma (HCC) cells (9). Taylor et al (10) reported that human tumor-derived epithelial cell adhesion molecule-positive exosomes could be detected in the blood by targeted miRNA expression profiling. Exosomes have been suggested as promising biomarkers of both ovarian (10) and lung cancer (11). These results suggested that exosomal miRNAs may serve as biomarkers for disease. Bala et al (12) demonstrated that the expression levels of circulating miRNAs were significantly upregulated in the plasma exosomes of patients with liver injuries, suggesting that circulating miRNAs may serve as biomarkers to differentiate between hepatocyte injury and inflammation. In addition, Murakami et al (13) demonstrated that the miRNA expression pattern in exosome-rich fractionated serum may serve as a biomarker for diagnosing the grade and stage of liver disease.

$\mathrm{HCC}$ is a major histological subtype of liver cancer, which presents as an aggressive tumor with poor prognosis (14). It is critical that the diagnosis of HCC be made at an early stage if effective therapeutic treatment is to be carried out. In circulating exosomes, the difference between miRNA expression levels pre- and post-resection of liver carcinoma allows for the identification of molecular markers for the diagnosis and predicted outcome of HCC. The first requirement for the detection of a reliable biomarker is the accurate measurement of the quantity of miRNA present in circulating exosomes. Currently, the stem-loop reverse transcription-quantitative polymerase chain reaction (RT-qPCR) is widely used to quantitatively analyze the levels of circulating miRNAs (15). Notably, the normalization of RT-qPCR data to stable reference genes (RGs) is critical for accurate miRNA quantification, due to variations that are not a direct consequence of the disease itself, including sample procurement, stabilization, RNA extraction, and target quantification (16). Therefore, the identification of optimal RGs that are stably expressed irrespective 
of treatment is necessary for the accurate normalization of exosomal miRNA quantification data.

In the present study, various RGs were used to normalize the circulating miRNA levels in patients of primary $\mathrm{HCC}$ with hepatitis B infection (HBV) following surgical treatment. The expression levels of 10 RGs were then examined, in accordance with the available literature (16-20). The most appropriate combination of RGs determined by each algorithm was subsequently used to assess the expression levels of miR-122, a known non-invasive biomarker of HCC (21). The results of the present study are the first, to best of our knowledge, to identify a set of RGs suitable for serum exosomal gene expression studies in liver carcinoma resection.

\section{Materials and methods}

Ethics statement. The present study was approved by the Medical Ethics Committee of the Second Affiliated Hospital of the Third Military Medical University (Chongqing, China), in accordance with the Helsinki Declaration. All patients provided written informed consent.

Serum preparation. Pre- and post-operative blood samples from patients with HCC were donated by the Second Affiliated Hospital of the Third Military Medical University (Table I). A tissue biopsy was performed prior to blood donation in order to diagnose HCC. All patients underwent complete resection without major morbidity or mortality.

The peripheral blood samples were collected in $5 \mathrm{ml}$ Vacutainer SST Plus Blood Collection Tubes (BD Biosciences, Franklin Lakes, NJ, USA). The samples were incubated at room temperature between $30 \mathrm{~min}$ and $2 \mathrm{~h}$. The tubes containing the samples were subsequently centrifuged at $1,500 \mathrm{x} g$ for $10 \mathrm{~min}$, and the sera were aliquoted and centrifuged again at $2,000 \mathrm{x} \mathrm{g}$, in order to completely remove any remaining cells. The sera were stored at $-80^{\circ} \mathrm{C}$ until further processing for exosome isolation.

Exosome preparation. A total of $250 \mu 1$ serum was mixed with $66 \mu$ l ExoQuick Exosome Precipitation Solution (SBI System Biosciences, Mountain View, CA, USA). The exosome isolation was performed in accordance with the manufacturer's instructions. Briefly, the samples were incubated at $4^{\circ} \mathrm{C}$ for $30 \mathrm{~min}$, followed by centrifugation at $17,760 \mathrm{x} \mathrm{g}$ for $2 \mathrm{~min}$. The protein-rich supernatant was then removed, and the exosome-rich pellet was retained for RNA extraction, electron microscopy and western blot analysis.

Transmission electron microscopy. Electron microscopy was performed on the serum exosome samples at the Biomedical Analysis Center, Third Military Medical University. The samples were prepared as described by Théry et al (22). Briefly, the exosomal fraction was mixed 1:1 with $4 \%$ paraformaldehyde in phosphate-buffered saline (PBS). The solution was subsequently placed onto formvar-carbon coated copper grids (Beijing Zhongjingkeyi Technology Co., Ltd., Beijing, China), and left to dry at room temperature for $20 \mathrm{~min}$. Following washing, the grids were fixed with $1 \% \mathrm{w} / \mathrm{v}$ glutaraldehyde in PBS, prior to being washed numerous times with distilled water. The samples were then contrasted using $4 \% \mathrm{w} / \mathrm{v}$ uranyl acetate and UA-Methylcellulose mix solution for $10 \mathrm{~min}$ on ice (22). The grid was dried at room temperature, and observed using a Tecnai 10 transmission electron microscope (FEI, Eindhoven, The Netherlands).

Western blot analysis. The exosome-rich pellet was resuspended in $1 \mathrm{X}$ radioimmunoprecipitation assay buffer(Beyotime Institute of Biotechnology, Shanghai, China), and protein concentration was quantified using a Bicinchoninic Acid assay (Beyotime Institute of Biotechnology, according to the manufacturer's instructions. The proteins ( $5 \mathrm{mg}$ ) were denatured by boiling in Laemmli sample buffer and separated by $12 \%$ SDS-PAGE, prior to being transferred onto a polyvinylidene fluoride membrane (Merck Millipore, Billerica, MA, USA). The blotting membrane was blocked with bovine serum albumin (Sigma-Aldrich, St. Louis, MO, USA) and incubated with CD63 and CD9 antibodies (1:1,000 dilution; cat. no. EXOAB-KIT-1; SBI System Biosciences) for $1 \mathrm{~h}$ at room temperature, followed by incubation with goat anti-rabbit horseradish peroxidase-conjugated secondary antibody (1:20,000 dilution; cat. no. EXOAB-KIT-1; SBI System Biosciences) for $1 \mathrm{~h}$ at room temperature. The blots were visualized using enhanced chemiluminescence (Thermo Fisher Scientific, Inc., Waltham, MA, USA).

Candidate RGs and primer design. A total of 10 candidate endogenous RGs were selected based on previous reports of their suitability for RT-qPCR associated with hepatopathy in tissues, serum, or plasma (16,17-20). Four of these genes were previously described as RGs for serum miRNA analysis: miR-26a, miR-221, miR-181c, and miR-451 (17,19,20). The remaining genes: miR-16, miR-103, miR-191, let-7a, 5S, and U6, were obtained from liver tissue studies.

The primer sequences of the candidate RGs, along with their corresponding bibliographic reference and amplicon parameters, are listed in Table II. The primers for 5S and U6 were purchased from Guangzhou RiboBio Co., Ltd. (Guangzhou, China). The NCBI (http://www.ncbi.nlm.nih.gov) and miRBase (http://www.mirbase.org/) databases were used to search for available gene sequences, and Primer 5 software (Premier Biosoft, Palo Alto, CA, USA) was used to design the primers. Primers were synthesized by Shanghai Bioengineering Co., Ltd. (Shanghai, China). The reaction conditions were optimized by determining the optimal annealing temperature and primer concentration.

RNA extraction and reverse transcription. Exosome-rich pellets were resuspended in $200 \mu \mathrm{l}$ PBS and lysed with $1 \mathrm{ml}$ QIAzole $^{\circledR}$ (Qiagen GmbH, Hilden, Germany). The RNA was isolated using the miRNeasy Serum/Plasma kit (Qiagen $\mathrm{GmbH}$ ), according to the manufacturer's instructions for liquid samples. Each RNA sample was then eluted in the same volume (normalization by volume) from a given volume of starting serum $(250 \mu \mathrm{l})$, and reverse transcribed to cDNA using the GoScript ${ }^{\mathrm{TH}}$ Reverse Transcription system (Promega Corporation, Madison, WI, USA).

$R T-q P C R$ reaction. The RT-qPCR reactions were performed in 96-well reaction plates using a StepOne Plus Real-Time PCR system (Applied Biosystems Life Technologies, Foster City, CA, USA). The final reaction volume was $20 \mu$, including 
Table I. Demographic and clinical features of patients used for microRNA expression analysis.

\begin{tabular}{|c|c|c|c|c|c|c|}
\hline \multirow[b]{2}{*}{ Variable } & \multicolumn{2}{|c|}{ Paired sample set $1(n=33)$} & \multirow[b]{2}{*}{ P-value } & \multicolumn{2}{|c|}{ Paired sample set $2(n=20)$} & \multirow[b]{2}{*}{ P-value } \\
\hline & $\begin{array}{c}\text { Pre-operative } \\
\text { patients }\end{array}$ & $\begin{array}{l}\text { Post-operative } \\
\text { patients }\end{array}$ & & $\begin{array}{l}\text { Pre-operative } \\
\text { patients }\end{array}$ & $\begin{array}{l}\text { Post-operative } \\
\text { patients }\end{array}$ & \\
\hline Average age (mean \pm SD) & $47.7 \pm 11.6$ & & & $46.1 \pm 12.1$ & & $0.675^{\mathrm{a}}$ \\
\hline Age (years), n (\%) & & & & & & $0.966^{\mathrm{b}}$ \\
\hline$<40$ & $6(18.2)$ & & & $4(20.0)$ & & \\
\hline $40-60$ & $21(63.6)$ & & & $12(60.0)$ & & \\
\hline$>60$ & $6(18.2)$ & & & $4(20.0)$ & & \\
\hline Gender, n (\%) & & & & & & $0.871^{\mathrm{b}}$ \\
\hline Male & $31(93.9)$ & & & $19(95.0)$ & & \\
\hline Female & $2(6.1)$ & & & $1(5.0)$ & & \\
\hline Tumor number, n (\%) & & & & & & $0.723^{\mathrm{b}}$ \\
\hline Single & $23(69.7)$ & & & $13(65.0)$ & & \\
\hline Multiple & $10(30.3)$ & & & $7(35.0)$ & & \\
\hline Tumor grade, $\mathrm{n}(\%)^{*}$ & & & & & & $0.805^{\mathrm{b}}$ \\
\hline I and II & $16(48.5)$ & & & $9(45.0)$ & & \\
\hline III and IV & $17(51.5)$ & & & $11(55.0)$ & & \\
\hline Average ALT $($ mean \pm SD) & $55.0 \pm 55.7$ & $110.7 \pm 86.6$ & $0.008^{\mathrm{c}}$ & $60.9 \pm 51.8$ & $108.1 \pm 85.0$ & $0.072^{\mathrm{c}}$ \\
\hline $\operatorname{ALT}(\mathrm{U} / \mathrm{l}), \mathrm{n}(\%)$ & & & $0.001^{\mathrm{b}}$ & & & $0.027^{\mathrm{b}}$ \\
\hline$<40$ & $16(48.5)$ & $4(12.1)$ & & $8(40.0)$ & $2(10.0)$ & \\
\hline $40-100$ & $14(42.4)$ & $17(51.5)$ & & $10(50.0)$ & $10(50.0)$ & \\
\hline$\geq 100$ & $3(9.1)$ & $12(36.4)$ & & $2(10.0)$ & $8(40.0)$ & \\
\hline
\end{tabular}

All subjects are hepatitis B virus patients with hepatocellular carcinoma. Paired sample set 1 was used for research of candidate reference genes, and paired sample set 2 was used for verification of the selected candidate reference genes. ${ }^{\mathrm{a}} \mathrm{t}$-test between two sets of data (paired sample set 1 vs. paired sample set 2); b two way $\chi^{2}$ test between two sets (paired sample set 1 vs. paired sample set 2) or two cohorts (pre-operative vs. post-operative) of data. c paired t-test between two cohorts of data (pre-operative vs. post-operative). ${ }^{*}$ Tumor grade was obtained according to the tumor node metastasis criteria. SD, standard deviation; ALT, alanine aminotransferase.

$10 \mu 1 \mathrm{SYBR}^{\circledR}$ Select Master Mix (Applied Biosystems Life Technologies), $250 \mathrm{nM}$ of each primer, and $2 \mu 1 \mathrm{cDNA}$ at a 1:4 dilution. The thermal cycling conditions included one cycle at $50^{\circ} \mathrm{C}$ for $2 \mathrm{~min}$, one cycle at $95^{\circ} \mathrm{C}$ for $2 \mathrm{~min}$, followed by 40 cycles of amplification at $95^{\circ} \mathrm{C}$ for $15 \mathrm{sec}$ and $60^{\circ} \mathrm{C}$ for $1 \mathrm{~min}$. The threshold cycle $(\mathrm{Cq})$ was calculated using SDS software 2.1 (Applied Biosystems Life Technologies), at a threshold value of 0.38 . Since the $\mathrm{Cq}$ values of all gene RT-negative controls $>36, \mathrm{Cq}$ values $<36$ were accepted for further experimentation.

PCR efficiency $(E)$. In order to calculate the efficiency of the RT-qPCR, standard curves were generated using 10-fold serial dilutions from a pool of cDNA (23). Duplicate standard curves were included in all RT-qPCR assays. The obtained individual $\mathrm{Cq}$ values were then plotted against the logarithm of the dilution factor, and both the Pearson's correlation coefficient (R), and PCR efficiency (E) for each assay were determined from the respective plots. Regression correlation coefficients $\left(\mathrm{R}^{2}\right)$ and efficiency (E) values were obtained from the GenEx 5 Standard software (BioMCC, Freising, Germany). In the present study, the Minimum Information for Publication of Quantitative Real-Time PCR Experiments guidelines (24) were followed, which promoted the effort for experimental consistency and transparency, and increased the reliability of the obtained results.
Statistical analysis. The expression stability of the candidate RGs was calculated using four widely used algorithms: geNorm (25), NormFinder (26), BestKeeper (27), and the comparative $\Delta \mathrm{Ct}$ method (28). These four methods were implemented using an online tool for evaluating reference gene expression (http://www.leonxie.com/referencegene.php) (29). The ranking of the RGs according to their stability was generated by each algorithm, and a series of continuous integers starting from 1 was assigned to each RG. The geomean of each gene weight across the four algorithms was subsequently calculated, following which the RGs were re-ranked according to geomean. The gene with the lowest geomean was considered to be the most stable RG (29). Statistical analyses were performed using SPSS 19.0 software (IBM SPSS, Armonk, NY, USA) followed by a paired t-test. $\mathrm{P}<0.05$ was considered to indicate a statistically significant difference. For the evaluation of statistical equivalence, a Student-Newman-Keuls test was used.

\section{Results}

Characterization of serum exosomes. Previous studies indicated that serum exosomes may enrich miRNAs, and thus more accurate and reproducible data may be obtained from exosome miRNA quantitative analysis $(5,13)$. The successful isolation of exosomes from serum is necessary for miRNA 


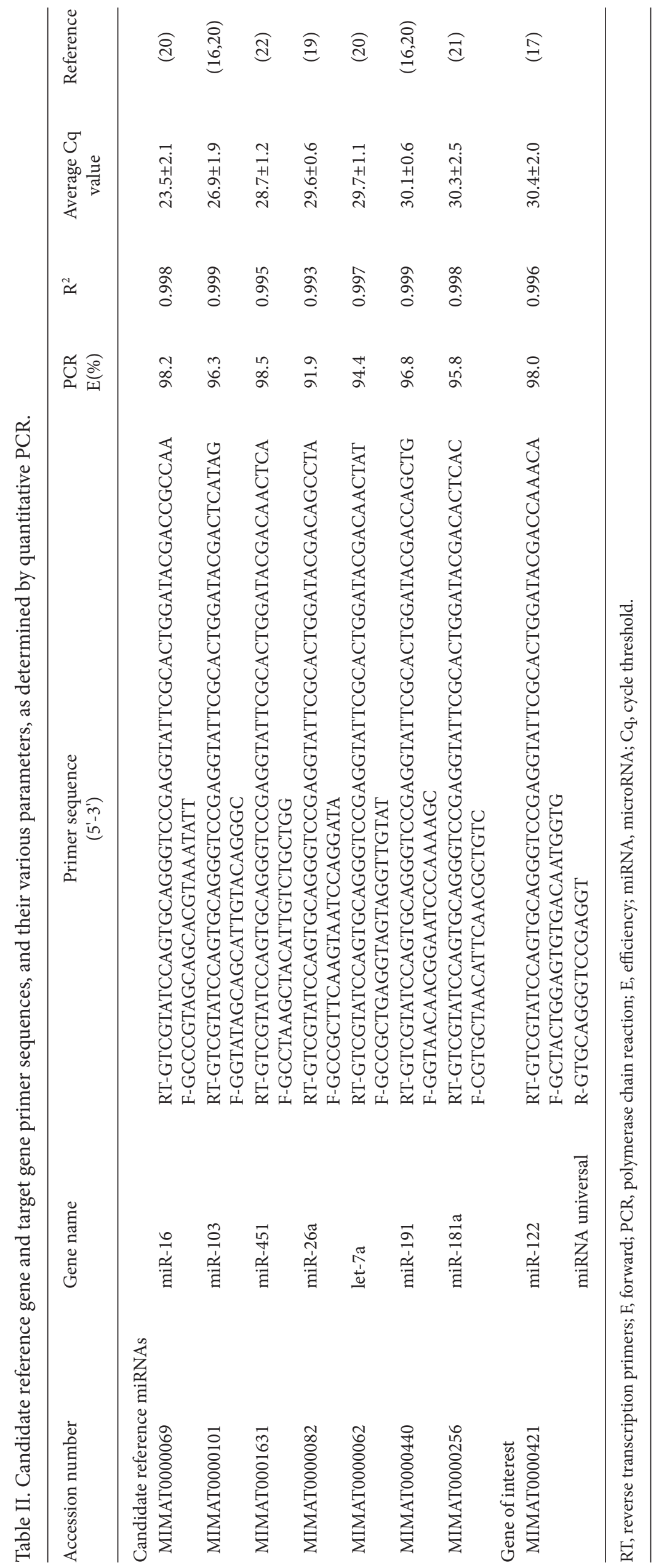


Table III. Expression levels of candidate reference genes.

\begin{tabular}{|c|c|c|c|c|c|c|}
\hline $\begin{array}{l}\text { Gene } \\
\text { name }\end{array}$ & $\begin{array}{l}\text { geNorm } \\
\text { M-value }\end{array}$ & $\begin{array}{l}\text { NormFinder } \\
\text { Stability value }\end{array}$ & $\begin{array}{c}\text { BestKeeper } \\
\mathrm{SD} \pm \mathrm{CP}\end{array}$ & $\begin{array}{c}\Delta \mathrm{Ct} \\
\text { Mean } \pm \mathrm{SD}\end{array}$ & $\begin{array}{l}\text { Comprehensive } \\
\text { gene stability }\end{array}$ & Ranking order ${ }^{\mathrm{a}}$ \\
\hline miR-221 & $0.67(1)$ & $0.67(2)$ & $0.52(2)$ & $1.50(2)$ & 1.68 & 1 \\
\hline let-7a & $0.67(1)$ & $0.73(3)$ & $0.46(1)$ & $1.56(3)$ & 1.73 & 2 \\
\hline miR-26a & $0.84(3)$ & $0.27(1)$ & $0.88(4)$ & $1.43(1)$ & 1.86 & 3 \\
\hline miR-103 & $1.13(5)$ & $1.22(6)$ & $0.85(3)$ & $1.81(6)$ & 4.82 & 4 \\
\hline miR-191 & $1.23(6)$ & $0.87(4)$ & $1.22(6)$ & $1.63(4)$ & 4.90 & 5 \\
\hline miR-181a & $1.03(4)$ & $1.46(7)$ & $0.92(5)$ & $1.92(7)$ & 5.60 & 6 \\
\hline miR-16 & $1.35(7)$ & $1.20(5)$ & $1.45(7)$ & $1.79(5)$ & 5.92 & 7 \\
\hline $5 \mathrm{~S}$ & $1.52(8)$ & $1.71(8)$ & $1.71(8)$ & $2.16(8)$ & 8.00 & 8 \\
\hline miR-451 & $1.69(9)$ & $2.16(9)$ & $1.99(9)$ & $2.46(9)$ & 9.00 & 9 \\
\hline U6 & $1.9(10)$ & $2.44(10)$ & $2.11(10)$ & $2.71(10)$ & 10.00 & 10 \\
\hline
\end{tabular}

${ }^{a}$ miRNAs are ranked according to gene stability as determined by RefFinder. The numbers in brackets represent the ranking values, regarded as a recommended final ranking. SD, standard deviation.

Table IV. Gene expression stability values and accumulated standard deviation (Acc. SD) analysis as determined by NormFinder.

\begin{tabular}{lccc}
\hline Gene name & Stability value & $\begin{array}{c}\text { NormFinder } \\
\text { Acc. SD value }\end{array}$ & Optimal RGs (n) \\
\hline miR-26a & $\mathbf{0 . 2 7}$ & $\mathbf{0 . 2 7}$ & $\mathbf{1}$ \\
miR-221 & 0.67 & 0.36 & 2 \\
let-7a & 0.73 & 0.34 & 3 \\
miR-191 & 0.87 & 0.34 & 5 \\
miR-16 & 1.20 & 0.36 & 6 \\
miR-103 & 1.22 & 0.36 & 7 \\
miR-181a & 1.46 & 0.39 & 8 \\
5S & 1.71 & 0.42 & 9 \\
miR-451 & 2.16 & 0.45 & 10 \\
U6 & 2.44 & & 5 \\
\hline
\end{tabular}

${ }^{a}$ Acc.SD value was calculated by equation A. The recommended optimal RG was marked in bold. SD, standard deviation; miR, microRNA; RG, reference gene.
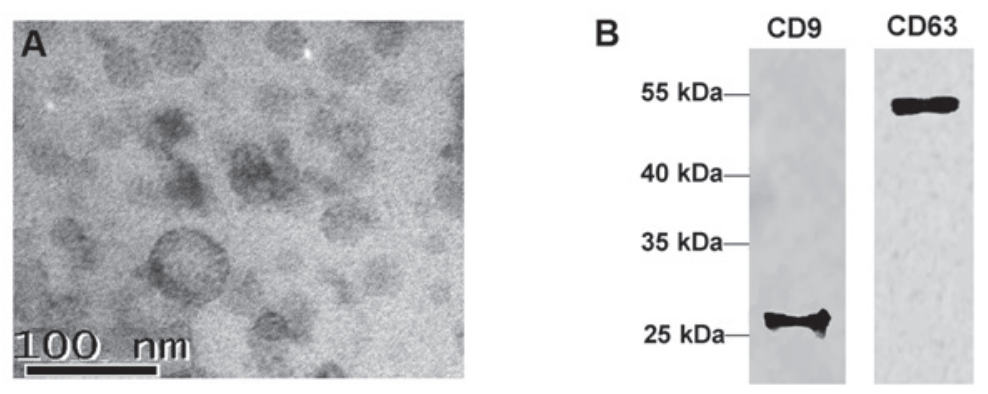

Figure 1. Identification of serum exosomes using transmission electron microscopy and western blot analysis. (A) Nanoscale structures in serum exosomes. Scale bar, $100 \mathrm{~nm}$. (B) The exosomes extracted from the serum were lysed with 1X radioimmunoprecipitation assay buffer. The exosomal tetraspanin protein markers CD63 and CD9 were then detected.

quantitative evaluation. In the present study, serum exosomes were obtained using an ExoQuick exosome precipitation solution. Electron microscopy indicated the presence of 30-100 $\mathrm{nm}$ spherical structures (Fig. 1A), consistent with previously reported exosome characteristics (30). These results were further confirmed with western blot analyses, using anti- bodies targeting two common exosomal markers, tetraspanin molecules CD63 and CD9, the results of which supported the endocytic origin of the vesicles (31-33) (Fig. 1B).

RT-qPCR assay validation. All PCR assays produced a single amplicon, as shown by the presence of a single marked 
increase on the melting curve. The negative controls did not contain template ("no-template control" (NTC)). The NTCs of 5S, miR-181c, and miR-191 were successfully detected, and their $\mathrm{Cq}$ values were $<36$. The PCRs displayed efficiency between 91.9 and $98.5 \%$ (Table II). PCR E values between 90 and $110 \%$ were also considered acceptable. The $\mathrm{R}^{2}$ values ranged between 0.993 and 0.999 (Table II).

Transcript profiles of RGs. The expression profiles of the 10 candidate RGs were assessed in paired sample set 1 (Fig. 2). The $\mathrm{Cq}$ values are represented for each transcript amplified from each biological replicate. The $\mathrm{Cq}$ values ranged between 19.25 (5S) and 35.75 (miR-181a). The Cq values for 5S and U6 were significantly different in the pre- and post-operative serum, $\mathrm{P}<0.001$, whereas no significant difference was detected for miR-16, miR-103, miR-451, miR-26a, miR-191, and miR-181a $(\mathrm{P}>0.05)$.

Gene expression stability analysis. In order to rank the stability of the tested genes, four algorithms: geNorm, NormFinder, BestKeeper, and the comparative $\Delta \mathrm{Ct}$ method, were used. These four methods evaluated the expression stability of the RGs, according to different variables. GeNorm provides an $\mathrm{M}$-value based on the average pairwise expression ratio. The most stable transcript has the lowest M-value, and RGs with $\mathrm{M} \leq 1.5$ were deemed to be stably expressed (25). According to this method, the M-values of the candidate RGs in 33 pairs of samples were $<1.5$, except for $5 \mathrm{~S}$, miR-451, and U6, suggesting that these were not reliable RGs. miR-221 and let-7a were the most stable genes, with an average expression stability of $\mathrm{M}=0.67$ (Table III),

NormFinder analyzes the expression stability of the RGs using linear scale quantitative data, and provides a stability value for each investigated gene. A higher stability value indicates a lower stability (26). In the present study, the NormFinder analysis identified miR-26a as the most stably expressed RG, with a stability value of 0.27 , followed by miR-221 (0.67) and let-7a (0.73) (Table III). The NormFinder algorithm was used to calculate the Accumulated Standard Deviation (Acc. SD) of the candidate RGs using the GenEx Standard software, according to equation $\mathrm{A}$. The lowest Acc. SD value indicates the optimal number of control genes. Based on the ranking of gene stability, the lowest Acc. SD value was determined to correspond to one optimal gene: miR-26a (Table IV).

$$
\mathrm{SD}(\operatorname{acc})=\frac{1}{n} \sqrt{\sum_{i=1}^{n} S D_{i}^{2}} \text { (Eq.A) }
$$

In the present study, the Acc. SD based on any given RG (n) was calculated as the geometric average of raw RG quantities, for any given gene (i).

BestKeeper determines gene stability according to SD, with a lower SD indicating a more stably expressed gene (27). The results of the BestKeeper analysis showed a high SD variation for miR-103, miR-16, 5S, miR-451 and U6. In the present study, let-7a was shown to be the most stable RG with $\mathrm{SD}=0.46$, followed by miR-221 with $\mathrm{SD}=0.52$ (Table III).

The comparative $\Delta \mathrm{Ct}$ method was also used to estimate the most stable RGs. The results of the $\Delta \mathrm{Ct}$ method were the same as those of the NormFinder analysis, with the three most stable RGs being miR-26a, miR-221, and let-7a (Table III).

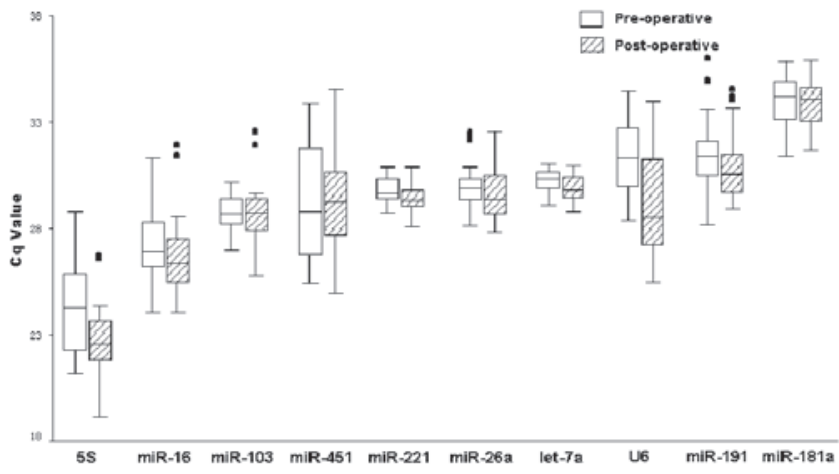

Figure 2. Expression levels of candidate reference genes in circulating exosomes. Reverse transcription-quantitative polymerase chain reaction was performed on the serum exosome samples. The boxplot indicates the interquartile range (IQR) and median. The whiskers indicate the highest and lowest $\mathrm{Cq}$ values that remain within $1.5 \mathrm{xIQR}$ of the 25 th and 75 th percentiles. The small circles indicate the outliers. miR, micro RNA; Cq, cycle threshold.

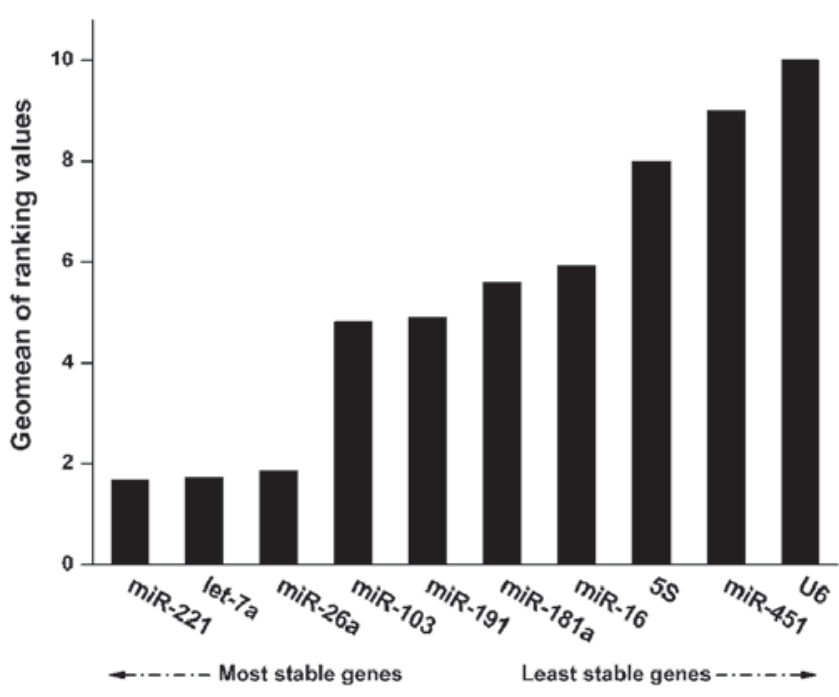

Figure 3. Gene stability of 10 candidate reference genes (RGs) in pre- and post-operative sera from patients with hepatocellular carcinoma. The geomean of the ranking values of gene stability (x-axis) are plotted against the 10 RGs (y-axis). Lower geomean values indicate stable gene expression.

Since there were differences in the results generated from the various software programs, normalization and integration of the data was necessary. RefFinder (http://www.leonxie. com/referencegene.php) is a web-based tool used to generate an overall ranking of candidate RGs (29). According to the output of RefFinder, the least stable RGs were U6, miR-451, and $5 \mathrm{~S}$. The three most stable RGs were miR-221, let-7a, and miR-26a (Fig. 3). Notably, the three algorithms also ranked miR-221, let-7a, and miR-26a as the most stable RGs, except in the case of miR-26a, which was ranked fourth most stable by BestKeeper (Table III). These results indicate the possible use of miR-221, let-7a, and miR-26a as stable RGs in liver carcinoma resection studies.

Impact of RGs on the expression levels of target genes. RT-qPCR assays were performed in order to further evaluate the expression patterns of the selected candidate RGs in the paired sample set 2 . Liver-specific miR-122 was chosen for 


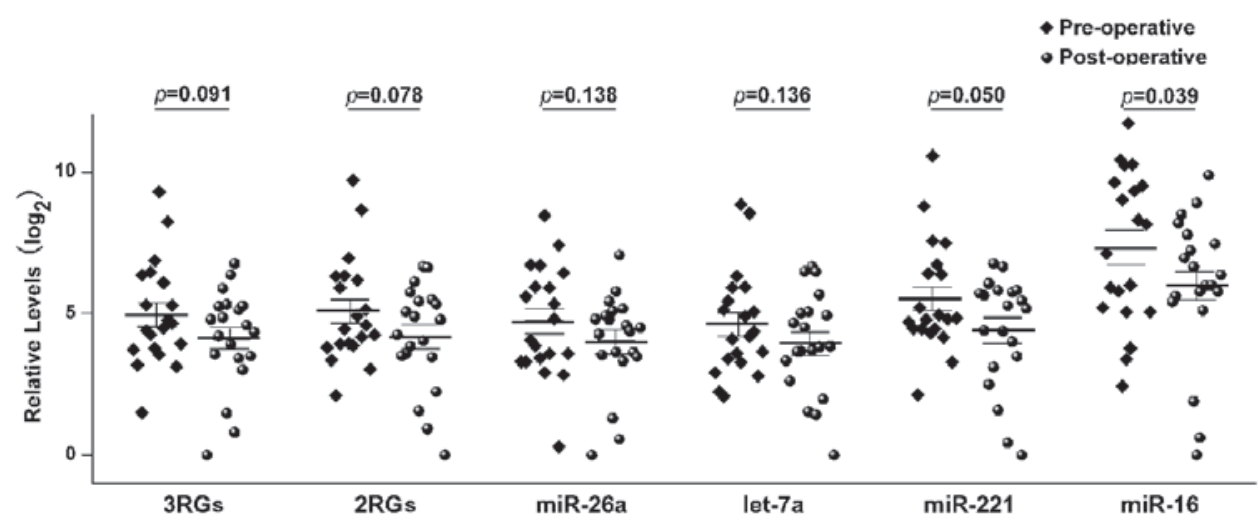

Figure 4. Relative microRNA (miR)-122 expression levels were measured by normalization to various reference genes (RGs). 3RGs represents miR-221, let-7a, and miR-26a, as recommended by RefFinder. 2RG represents miR-221 and let-7a as recommended by geNorm. miR-26a was recommended by NormFinder. let-7a, miR-26a, or miR-16 were used as single RGs for normalization.

analysis, due to its higher expression levels in the sera of patients with HCC following surgery (21). The miR-122 expression level data were normalized (Fig. 4) using the RefFinder recommended combination of miR-221, let-7a, and miR-26a (3RGs), and using the geNorm recommended combination of miR-221 and let-7a (2RGs). miR-26a was recommended by NormFinder according to the lowest Acc. SD value. In addition, the data was further normalized using a single gene; let-7a, miR-221, or miR-16. Although BestKeeper and geNorm did not recommend miR-16 as a suitable RG, it is nevertheless frequently used in expression studies $(34,35)$. The fold changes in serum exosomal miR-122 between the various groups were calculated using the GenEx software. In the present study, normalization with miR-16 indicated that serum exosomal miR-122 was downregulated following liver cancer surgery, whereas normalization using other RGs, except for miR-221, did not indicate differential expression of the target miRNA. In addition, normalization of miR-122 expression by miR-221, a statistically significant value of $\mathrm{P}<0.05$ was generated. These results suggest that the reliability of miR-221 as a RG needs to be confirmed in further studies with larger sample sizes. In conclusion, the use of miR-16 for data normalization generated identical results to that of Qi et al (21). A previous study demonstrated that the expression levels of miR-122 were significantly reduced in post-operative serum samples, as compared with pre-operative samples (21). However, other normalization approaches used based on the recommendations of the present study indicated that hepatic surgery did not change the expression levels of miR-122 in serum exosomes.

\section{Discussion}

Selecting an appropriate RG is important for gene expression analysis, as the use of an inappropriate RG may lead to false experimental conclusions $(36,37)$. Therefore, one or more appropriate RGs need to be selected depending on the experimental conditions.

The evaluation of the expression stability of candidate RGs in serum exosomes from individuals with liver carcinoma has yet to be reported. The use of optimal RGs would contribute significantly to the accurate identification of biomarkers and predictive factors used to diagnose HCC, and to predict early post-operative relapse in patients with HCC. Based on previous studies, 10 commonly used RGs were selected for gene stability analysis (16-20). In order to evaluate the average expression stability of the RGs, four algorithms (geNorm, NormFinder, BestKeeper and the comparative $\Delta \mathrm{Ct}$ method), were used.

A conventional statistical test was initially conducted in order to evaluate the expression dispersion of each gene in preand post-operative patients with HCC (Fig. 2). The stability of the candidate RGs in the various sample sets were subsequently analyzed using the four algorithms. The pair-wise comparison approach methods (geNorm and BestKeeper) selected the most suitable RGs based on the variation of expression ratios between the genes across the sample sets. Both methods generated similar rankings, and both considered miR-221 and let-7a to be the most suitable RGs for normalization (Table III). However, the rankings differed significantly for miR-26a, miR-103, and miR-181a. This may be due to the fact that geNorm excludes genes with differences in expression in the subgroups, but includes pairs of co-regulated genes based on their similar expression profiles (26). Therefore, NormFinder and the comparative $\Delta \mathrm{Ct}$ method were used in order to eliminate the effects of co-regulation, and to evaluate the RGs from all aspects. These two algorithms generated the same RG rankings based on the calculation of gene stability. miR-26a was considered the most suitable RG for serum exosomal miRNA expression studies in patients with HCC following surgery. Finally, using the web-based comprehensive tool RefFinder, miR-221, let-7a, and miR-26a were determined to be the most stable RGs. Furthermore, the results of the present study demonstrated that $5 \mathrm{~S}, \mathrm{miR}-451$, and U6 were not suitable as housekeeping genes for the present experimental setup. With regards to miR-16, geNorm and BestKeeper indicated that its stability value exceeded that required for a stable RG, making it unreliable for miRNA expression analysis.

The effects of the normalization strategies were further illustrated by the accuracy of the RT-qPCR results. miR-122 serum expression levels have previously been shown to be downregulated in the serum of post-operative patients with HCC (21). The results of the present study revealed that circulating miR-122 was significantly reduced in the post-operative serum samples when miR-16 was used as an RG, in a similar 
manner to that of Qi et al (21). However, when the data were normalized to miR-221, let-7a, and miR-26a combined, miR-221 and let-7a combined, or miR-26a alone, as recommended by the results of the present study, a statistically significant difference in miR-122 expression was not detected. Therefore, rigorous validation of RG suitability is required, as different normalization controls were shown to significantly influence serum expression levels of miR-122, despite the fact that the miRNA samples were donated by patients of the same descent with the same disease. A previous study reported that normalization with miR-16 resulted in significantly higher miR-122 expression levels detected in patients with HCC, as compared with HBV-infected individuals (21). In addition, when miR-181a, miR-181c, and miR-122 were used as RGs, miR-122 expression was higher in patients with HCC, as compared with HBV-infected controls (19). However, $\mathrm{Qu}$ et al (38) demonstrated that the serum expression levels of miR-16 were significantly lower in patients with HCC, suggesting that miR-16 itself may act as a novel biomarker for HCC. Therefore, the use of miR-16 as a normalizer of target miRNA expression levels may result in erroneous results, confirming the results of the present study that miR-16 is not a suitable RG candidate. The present study also demonstrated that systematically selected RGs should offer more appropriate normalization than miR-221.

The results of the present study indicated that the accurate selection of reliable RGs is a prerequisite for the correct measurement of serum exosomal miRNA expression levels by RT-qPCR. The following RGs: miR-221, let-7a, and miR-26a, were the most stably expressed genes of the present study. Furthermore, due to the technical and economic advantages of using a smaller number of RGs, miR-221 and let-7a combined, or miR-26a alone may be used as optimal RGs in order to detect a single target miRNA.

\section{Acknowledgements}

The present study was supported by a grant from the National Natural Science Foundation of China (grant no. 81201356). The authors of the present study are thankful for the valuable comments from members of the Department of Clinical Laboratory (Chongqing, China).

\section{References}

1. Ambros V: The functions of animal microRNAs. Nature 431: 350-355, 2004

2. Li W, Chen YQ, Shen YB, et al: HIF-1 $\alpha$ knockdown by miRNA decreases survivin expression and inhibits A549 cell growth in vitro and in vivo. Int J Mol Med 32: 271-280, 2013.

3. Vlassov AV, Magdaleno S, Setterquist R and Conrad R: Exosomes: Current knowledge of their composition, biological functions, and diagnostic and therapeutic potentials. Biochim Biophys Acta 1820: 940-948, 2012.

4. Giusti I, D'Ascenzo S and Dolo V: Microvesicles as potential ovarian cancer biomarkers. BioMed Res Int 2013: 703048, 2013.

5. Gallo A, Tandon M, Alevizos I and Illei GG: The majority of microRNAs detectable in serum and saliva is concentrated in exosomes. PLoS One 7: e30679, 2012

6. Michael A, Bajracharya SD, Yuen PS, et al: Exosomes from human saliva as a source of microRNA biomarkers. Oral Dis 16: 34-38, 2010.

7. Keller S, Ridinger J, Rupp AK, Janssen JW and Altevogt P: Body fluid derived exosomes as a novel template for clinical diagnostics. J Transl Med 9: 86, 2011.
8. Valadi H, Ekström K, Bossios A, Sjöstrand M, Lee JJ and Lötvall JO: Exosome-mediated transfer of mRNAs and microRNAs is a novel mechanism of genetic exchange between cells. Nat Cell Biol 9: 654-659, 2007.

9. Wahlgren J, De L Karlson T, Brisslert M, et al: Plasma exosomes can deliver exogenous short interfering RNA to monocytes and lymphocytes. Nucleic Acids Res 40: e130, 2012.

10. Taylor DD and Gercel-Taylor C: MicroRNA signatures of tumor-derived exosomes as diagnostic biomarkers of ovarian cancer. Gynecol Oncol 110: 13-21, 2008.

11. Rabinowits G, Gerçel-Taylor C, Day JM, Taylor DD and Kloecker GH: Exosomal microRNA: A diagnostic marker for lung cancer. Clin Lung Cancer 10: 42-46, 2009.

12. Bala S, Petrasek J, Mundkur S, et al: Circulating microRNAs in exosomes indicate hepatocyte injury and inflammation in alcoholic, drug-induced, and inflammatory liver diseases. Hepatology 56: 1946-1957, 2012.

13. Murakami Y, Toyoda H, Tanahashi T, et al: Comprehensive miRNA expression analysis in peripheral blood can diagnose liver disease. PLoS One 7: e48366, 2012.

14. Tang ZY, Ye SL, Liu YK, et al: A decade's studies on metastasis of hepatocellular carcinoma. J Cancer Res Clin Oncol 130: 187-196, 2004.

15. Lin Q, Mao W, Shu Y, et al: A cluster of specified microRNAs in peripheral blood as biomarkers for metastatic non-small-cell lung cancer by stem-loop RT-PCR. J Cancer Res Clin Oncol 138: 85-93, 2012

16. Peltier HJ and Latham GJ: Normalization of microRNA expression levels in quantitative RT-PCR assays: Identification of suitable reference RNA targets in normal and cancerous human solid tissues. RNA 14: 844-852, 2008.

17. Zhu HT, Dong QZ, Wang G, et al: Identification of suitable reference genes for qRT-PCR analysis of circulating microRNAs in hepatitis B virus-infected patients. Mol Biotechnol 50: 49-56, 2012.

18. Lardizábal MN, Nocito AL, Daniele SM, Ornella LA, Palatnik JF and Veggi LM: Reference genes for real-time PCR quantification of microRNAs and messenger RNAs in rat models of hepatotoxicity. PLoS One 7: e36323, 2012.

19. Xu J, Wu C, Che X, et al: Circulating microRNAs, miR-21, miR-122, and miR-223, in patients with hepatocellular carcinoma or chronic hepatitis. Mol Carcinog 50: 136-142, 2011.

20. Blondal T, Jensby Nielsen S, Baker A, et al: Assessing sample and miRNA profile quality in serum and plasma or other biofluids. Methods 59: S1-S6, 2013.

21. Qi P, Cheng SQ, Wang H, Li N, Chen YF and Gao CF: Serum microRNAs as biomarkers for hepatocellular carcinoma in Chinese patients with chronic hepatitis B virus infection. PLoS One 6: e28486, 2011.

22. Théry C, Amigorena S, Raposo G and Clayton A: Isolation and characterization of exosomes from cell culture supernatants and biological fluids. Curr Protoc Cell Biol Chapter 3: Unit 3.22, 2006.

23. Pfaffl MW: A new mathematical model for relative quantification in real-time RT-PCR. Nucleic Acids Res 29: e45, 2001.

24. Bustin SA, Benes V, Garson JA, et al: The MIQE guidelines: Minimum information for publication of quantitative real-time PCR experiments. Clin Chem 55: 611-622, 2009.

25. Vandesompele J, De Preter K, Pattyn F, et al.: Accurate normalization of real-time quantitative RT-PCR data by geometric averaging of multiple internal control genes. Genome Biol 3: RESEARCH0034, 2002.

26. Andersen CL, Jensen JL and Ørntoft TF: Normalization of real-time quantitative reverse transcription-PCR data: A model-based variance estimation approach to identify genes suited for normalization, applied to bladder and colon cancer data sets. Cancer Res 64: 5245-5250, 2004.

27. PfafflMW,Tichopad A,PrgometCandNeuvians TP:Determination of stable housekeeping genes, differentially regulated target genes and sample integrity: BestKeeper - Excel-based tool using pair-wise correlations. Biotechnol Lett 26: 509-515, 2004.

28. Silver N, Best S, Jiang J and Thein SL: Selection of housekeeping genes for gene expression studies in human reticulocytes using real-time PCR. BMC Mol Biol 7: 33, 2006.

29. Xie F, Xiao P, Chen D, Xu L and Zhang B: miRDeepFinder: A miRNA analysis tool for deep sequencing of plant small RNAs. Plant Mol Biol 80: 75-84, 2012.

30. Silverman JM and Reiner NE: Exosomes and other microvesicles in infection biology: Organelles with unanticipated phenotypes. Cell Microbiol 13: 1-9, 2011. 
31. Pols MS and Klumperman J: Trafficking and function of the tetraspanin CD63. Exp Cell Res 315: 1584-1592, 2009.

32. Mathivanan S and Simpson RJ: ExoCarta: A compendium of exosomal proteins and RNA. Proteomics 9: 4997-5000, 2009.

33. Mathivanan S, Ji H and Simpson RJ: Exosomes: Extracellular organelles important in intercellular communication. J Proteomics 73: 1907-1920, 2010.

34. Song J, Bai Z, Han W, et al: Identification of suitable reference genes for qPCR analysis of serum microRNA in gastric cancer patients. Dig Dis Sci 57: 897-904, 2012.

35. Li J, Wei H, Li Y, Li Q and Li N: Identification of a suitable endogenous control gene in porcine blastocysts for use in quantitative PCR analysis of microRNAs. Sci China Life Sci 55: 126-131, 2012.
36. Bustin SA and Nolan T: Pitfalls of quantitative real-time reverse-transcription polymerase chain reaction. J Biomol Tech 15: 155-166, 2004

37. Dheda K, Huggett JF, Chang JS, et al.: The implications of using an inappropriate reference gene for real-time reverse transcription PCR data normalization. Anal Biochem 344: 141-143, 2005.

38. Qu KZ, Zhang K, Li HR, Afdhal NH and Albitar M: Circulating microRNAs as biomarkers for hepatocellular carcinoma. J Clin Gastroenterol 45: 355-360, 2011. 